\title{
Effectiveness of a Tailored Work-Related Support Intervention for Patients Diagnosed with Gastrointestinal Cancer: A Multicenter Randomized Controlled Trial
}

\author{
A. C. G. N. M. Zaman ${ }^{1}$ - K. M. A. J. Tytgat ${ }^{2}$ J. H. G. Klinkenbij| $\left.\right|^{3,4}$ - F. C. den Boer ${ }^{5}$ - M. A. Brink ${ }^{6}$. J. C. Brinkhuis ${ }^{7}$ \\ D. J. Bruinvels ${ }^{7}$. L. C. M. Dol ${ }^{8}$ - P. van Duijvendijk ${ }^{3}$ - P. H. J. Hemmer ${ }^{9}$ B. Lamme ${ }^{10}$ - O. J. L. Loosveld ${ }^{11}$ - M. M. Mok ${ }^{12}$.

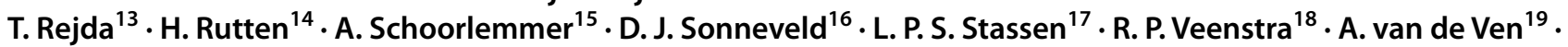 \\ E. R. Velzing ${ }^{20}$ - M. H. W. Frings-Dresen ${ }^{1}$ - A. G. E. M. de Boer ${ }^{1}$
}

Published online: 2 September 2020

(c) The Author(s) 2020

\begin{abstract}
Purpose The aim of this research was to study the effectiveness on return to work (RTW) of an early tailored work-related support intervention in patients diagnosed with curative gastrointestinal cancer. Methods A multicenter randomized controlled trial was undertaken, in which patients were assigned randomly to the intervention or the control group (usual care). The intervention encompassed three psychosocial work-related support meetings, starting before treatment. Five self-reported questionnaires were sent over twelve months of follow-up. Primary outcome was days until RTW (fulltime or partial) and secondary outcomes included work status, quality of life, work ability, and work limitations. Descriptive analysis, KaplanMeier analysis, relative risk ratio and linear mixed models were applied. Results Participants $(\mathrm{N}=88)$ had a mean age of 55 years; $67 \%$ were male and the most common cancer type was colon cancer (66\%). Of the participants, 42 were randomized to the intervention group. The median time from sick leave until RTW was 233 days (range 187-279 days) for the control group, versus 190 days (range 139-240 days) for the intervention group ( $\log$-rank $\mathrm{p}=0.37$ ). The RTW rate at twelve months after baseline was $83.3 \%$ for the intervention group and $73.5 \%$ for the control group. Work limitations did statistically differ between the groups over time ( $\mathrm{p}=0.01)$, but quality of life and work ability did not. Conclusion Patients in the intervention group seem to take fewer days to RTW, albeit not to a statistically significant extent.

Trial registration Trial NL4920 (NTR5022) (Dutch Trial Register https://www.trialregister.nl)
\end{abstract}

Keywords Return to work $\cdot$ Neoplasms $\cdot$ Randomized controlled trial $\cdot$ Vocational rehabilitation $\cdot$ Rehabilitation research

\section{Introduction}

The diagnosis of cancer has a major effect on a patient's life. Daily functioning in normal activities, including work, is affected by (long-term) physical and mental health problems [1], e.g. fatigue [2-4], and cognitive problems [3, 5] due to the malignancy and its treatment. One of the challenges for patients treated with curative intent is their return to work (RTW) after the treatment. Work plays an important,

M. H. W. Frings-Dresen and A. G. E. M. de Boer have contributed equally to this paper as principal investigators.

A. C. G. N. M. Zaman

a.c.zaman@amsterdamumc.nl

Extended author information available on the last page of the article positive role in people's lives. It contributes to better quality of life [6], it gives the feeling of participating as a 'normal' individual i.e. structures everyday life [7-9]. Furthermore, work can provide a better self-image and esteem [10], it contributes to social inclusion [8] and offers a source of financial security [11]. This is becoming even more important as the number of patients diagnosed with cancer in the working population is rising, age at diagnosis is falling (due to screening programs) and with advanced treatments survival rates increasing.

Previous research has shown that patients experience work-related problems from the moment of diagnosis [12] and appreciate work-related information in the early stages of their cancer treatment [12-14]. Moreover, a longer absence from work is associated with a reduced probability of RTW $[15,16]$. The health problems like fatigue and 
cognitive problems contribute to the RTW process and could even impede the work process. In the clinical setting, however, 'work' is not yet a standardized item discussed either in an early phase of diagnosis $[17,18]$ or later, once rehabilitation starts [19]. Yet it is relevant to prepare patients for the impact of cancer and its treatment on sick leave by giving them timely information about work-related issues, since this should enhance their self-empowerment to solve work-related problems. Therefore, the work-related information should be tailored to the patients' needs and include for example; how to deal with openness about the diagnosis to colleagues and/or employer, information about the Act when patient is reporting for sickness absence, and disease- and treatment-specific factors in relation to work must be discussed [20]. There is an increasing focus on psychosocial and other forms of support, such as physical activity $[5,21]$ for patients diagnosed with cancer in the occupational and oncological context [5, 22]. Moreover, some intervention studies have focused on work- related support [23-25].

Currently, though, there is still no intervention that features early work-related support and is tailored to the needs of the patient. It was for this reason that we initiated the GIRONA study (Gastro-Intestinal cancer patients Receiving Occupational support Near and After diagnosis), providing tailored work-related support for patients diagnosed with GI cancer [20]. The intention of this intervention was to start informing patients about work-related issues in an early phase of their diagnosis and to support them if they experienced work-related problems. The intervention was tailored to the severity of their individual work-related problems and delivered by specific supporting healthcare professional.

Our aim was to study the effectiveness of the GIRONA intervention by performing a multicenter randomized controlled trial with a follow-up period of twelve months. The intervention was compared with the 'usual' clinical care of patients diagnosed with curative GI cancer, which involves no work-related support. The hypothesis was that offering tailored work-related support early in the clinical diagnostic phase would lead to enhancement of RTW and therefore result into fewer days of sick leave.

\section{Methods}

The CONSORT statement $[26,27]$ was used to structure the trial methods and for reporting its results. In this paper we outline the tailored work-related support intervention itself; for background details, we refer readers to previous published articles on its design [20] and development [28].

\section{Trial Design}

The study was designed as a parallel, multicenter randomized controlled trial (RCT) with a follow-up period of twelve months. The intervention included tailored workrelated support for the intervention group, which was compared with 'usual care' received by the control group. The allocation ratio between the two groups was 1:1.

Supplementary to the previously published eligibility criteria [20], we adjusted one inclusion criterion. At the beginning of the study, this criterion was formulated as "patients were on sick leave at the time of diagnosis, i.e. the moment of study participation. As the study progressed, however, we noticed that patients were not always on sick leave yet at the moment of diagnosis, i.e. their first moment in the clinical setting. We therefore adjusted this inclusion criterion to assume that patients would be on sick-leave once their treatment started.

\section{Ethical Statements}

The Medical Research Involving Human Subjects Act was applied for this RCT study, as confirmed by the Medical Ethics Review Committee of the Academic Medical Center (AMC), Amsterdam (registration number W14_248\#14.17.0300). All participating hospitals approved local permission to start the study.

\section{Participants}

The GIRONA study was conducted in 16 hospitals in the Netherlands. We started the study with fewer hospitals as described in the protocol [20], because several hospitals were pending for the local medical ethical approval to participate. The participants in the trial included healthcare professionals and patients. The oncologist and the oncological GI nurses recruited the patients. Oncological GI nurses and oncological occupational physicians (OOPs) were the professionals providing the work-related support. The OOP is a specialized occupational physician trained in supporting patients who are diagnosed with cancer and in dealing with work-related issues. The OOP works within the clinical setting or outpatient clinic. However, those OOPs are not yet officially incorporated in cancer care [29]. The patients included were persons diagnosed with a primary GI cancer (i.e. a malignancies in the digestive tract system, ranging from the esophagus to the colorectum) that was treatable with curative intent (all treatments were included). They were between 18-63 years of age and in paid work (including temporary and flexible contracts and self-employed) at the moment of diagnosis. It was originally intended that they 
would have to be on sick leave, either full or partial, at the time of diagnosis, but as mentioned this latter inclusion criterion was revised. Patients who had a severe mental disorder or other severe co-morbidities (observed by the nurses who included eligible patients, not from the self-reported patient questionnaire), were excluded. If there was a doubt about co-morbidities, this was discussed between research group and the nurse who included the patients.

\section{Enrollment and Informed Consent Procedure}

The wards included were mainly surgical departments. One internal medicine department included patients, however, patients treated, for example, with neo-adjuvant therapy were discussed in the multidisciplinary meeting and therefore those patients could also be included by the oncological nurses. The nurses who enrolled the participants were also the nurses who provided the work-related support. Nurses were trained with a two-hour training before the start of the study, see for more details the design study [20].

Patients were asked to participate at the hospital where they were receiving their treatment. The oncological physician or nurse checked each patient's eligibility by assessing the inclusion and exclusion criteria during their first visit to the hospital, then provided the patient with a brief explanation of the study. The patient was asked whether the researcher could contact them by telephone. If the patient agreed, they signed a specific informed consent form for telephone contact. They were also given a folder with an information leaflet, contact details and the informed consent form.

A member of the research team [AZ, SvH, FD, or LJ] then called the patient to provide more details about the study and to answer any outstanding questions. Written informed consent was obtained from all individuals who participated in the GIRONA study.

\section{The GIRONA Intervention}

We developed this intervention [28] in close collaboration with a variety of healthcare professionals, including oncological physicians, oncological occupational physicians, oncological nurses and patients diagnosed with GI cancer. The tailored work-related support intervention encompassed three individual meetings of psychosocial work-related support. The first was to inform patients about work during and after treatment, to identify any work-related problems they might already have and to make a plan for their RTW or to stay in work. The first meeting was scheduled before the treatment began, the second a maximum of six months after the first and the third (if necessary) a maximum of nine months after the first.
Each meeting lasted approximately $30 \mathrm{~min}$. The first and second meeting needed to be face-to-face as per protocol; the third could also be conducted by telephone. Topics discussed were the importance of work, contact with the work environment, transparency about their diagnosis with employer and/or colleagues, and the process of reporting sick under Dutch law.

The intervention itself was split into three types of workrelated support (A, B and C); this was in order to respond to the patients' individual needs, since work-related problems can differ in severity. Based on contributing factors to such problems as described in a decision diagram [28]. For example in support A; fatigue, pain and lack of support from family and friends, in support B; lack of support in work environment, neuropsychological problems and in support $\mathrm{C}$; a combination of factors. These factors were assessed in the patients' baseline questionnaire (T0). Based on the answers and the decision diagram, the researcher referred the patient to the tailored type of support, respectively A, $\mathrm{B}$ or C. Within each of these, the kind of healthcare professional assigned to provide supportive work-related care was tailored to the severity of the patient's work-related problems and the healthcare professional takes the kind of work of the patient into account. In support type A this was an oncology nurse, in type $\mathrm{B}$ an OOP, and in type $\mathrm{C}$ a multidisciplinary team (including at least an oncology nurse, the treating physician, and an OOP). Details of the process evaluation are published separately [30].

\section{Usual Care}

Standard or 'usual' psychosocial care is defined as care provided by the oncological nurse or oncological physician/ surgeon, focusing on the treatment itself and related problems, e.g. pain or wound care. In general, work-related issues associated with the cancer diagnosis and treatment are not discussed.

\section{Measures}

All the outcome measures were obtained from five selfreported patient questionnaires, completed on paper or in digital form at baseline (T0), three months (T1), six months (T2), nine months (T3), and twelve months (T4).

\section{Primary Outcome}

The primary outcome was the number of days from sick leave until RTW (either fulltime or partial). Reflecting the Dutch social security system's regulations in respect of sick leave, RTW was defined as the patient having returned to work for at least four successive weeks (regardless of their contract hours). The period between taking sick leave and 
RTW was measured using the dates (first day of leave and RTW) provided by the patient in the five questionnaires. When no first date of sick leave was reported, the fifteenth of the month of diagnosis was used; when no date of RTW was reported, the date of completing the questionnaire was used.

\section{Secondary Outcomes}

Secondary outcome measures included employment status (RTW yes/no) at twelve months after baseline, as well as quality of life, work ability, and work limitations as assessed at each measurement point.

Quality of life was assessed using Short Form 12 (SF-12) $[31,32]$, covering twelve items (Dutch version). Questions inquired about the patient's functional status, including their physical and social functioning, physical and emotional constraints, and general view of their own health. Their answers were converted into two summary norm-based scale scores, for physical and mental health respectively. In both cases, the higher the score, the better the respondent's functioning, with a mean score of 50 (SD 10) in the general population [32]. SF-12 has been shown to be a valid and reliable instrument [31, 32]. Quality of life was additionally assessed with the EORTC QLC-C30, but we cannot report the results because of printing issues in the patient questionnaires.

Work ability was assessed using the first question from the Work Ability Index (WAI) [33] questionnaire (Dutch version), concerning current work ability compared with lifetime best ability. The answer format was a ten-point scale; the higher the score, the better the respondent's work ability $(0=$ not currently able to work at all; $10=$ work ability at its best). The WAI has been assessed as having good levels of reliability and validity [34].

Work limitation was measured using the Work Limitation Questionnaire (WLQ) [35], with a five-point scale evaluating the respondent's functional limitations. The WLQ covers 25 items, aggregated into four subscales (time management, physical demands, mental-interpersonal demands, and output demands). The scale ranges from 0 to 100, with higher scores indicating more work limitations over the previous two weeks $(0=$ never limited; $100=$ limited all the time $)$. The English version of the WLQ has been shown to be valid and reliable when used for cancer survivors [36], has been translated into Dutch, and is valid and reproducible at group level among cancer survivors [37].

\section{Prognostic Factors}

The following prognostic factors on RTW were taken into account: age, gender, marital status (married/cohabiting, single, divorced/widowed), educational attainment (low, intermediate, high), diagnosis, treatment type (not all patients had entered treatment at $\mathrm{T} 0$, therefore $\mathrm{T} 1$ was used), fatigue, depression, and cognitive functioning. Fatigue was measured using the Multidimensional Fatigue Inventory questionnaire (MFI), with 20 items divided into five subscales; subscale scores range from 4 to 20 , with higher scores indicating greater fatigue [38]. Depression was measured using the scale developed by the Center for Epidemiologic Studies for depression (CES-D), with 20 items; scores range from 0 to 60 , with higher scores indicating greater depressive symptoms measured for the past week [39]. Cognitive functioning was measured using the Cognitive Symptom ChecklistWork, Dutch Version (CSC-W DV), with 19 items; scores range from 0 to 100 , with higher scores indicating more cognitive symptoms or limitations [40].

\section{Descriptive Factors}

The descriptive factors included: main wage earner (yes/ no), employment status (permanent, temporary, or selfemployed), years in current position, and years in paid work. Another was workload perception, measured using the 'physical workload' subscale from the Perception and Judgment of Work questionnaire (VBBA), with seven items; scores range from 0 to 21, with higher scores indicating a higher physical workload [41]. Yet another was importance of work, measured using a Visual Analogue Scale (VAS); scores range from not important to most important. Also recorded was the use of other work-related co-interventions: a reintegration agency, reintegration coach, rehabilitation program, or support from other healthcare professionals.

\section{Sample Size}

Data from a large occupational health service in the Netherlands was used [42] to estimate the RTW percentage in the control group at $63 \%$. To estimate the RTW percentage in the intervention group, we referred to an earlier study in which cancer patients received a work-related support intervention including an educational leaflet and enhanced communications on the part of their attending and occupational physicians [43]. The RTW percentage in that study was $89 \%$ after twelve months; however, the median time between beginning treatment and enrollment was 42 days. Because our study started earlier, at the moment of diagnosis, we corrected this estimate with data from two previous studies conducted at our own hospital $[44,45]$. The RTW percentage in these was approximately $8 \%$ in the first 42 days. Hence, the RTW for the intervention group in our study was calculated as $81 \%(=89 \%-8 \%)$.

The required sample size was then calculated using the power and sample-size calculation program nQuery Advisor 7.0. A power of $80 \%$ and a $p$ value of $<0.05$ indicated that we should include a total of 216 patients, with a follow-up period of twelve months, to indicate a difference of $81 \%$ 
(intervention) versus 63\% RTW (care as usual). Allowing for a $20 \%$ loss to follow-up and a $10 \%$ one-year mortality rate, 309 patients should therefore be included [20].

\section{Randomization}

Once the researcher received their completed baseline questionnaire, the patient was randomized. Using a computerized web-based randomization program, ALEA [46], this randomization was conducted centrally at the AMC by the research team [AZ, SvH, FD, LJ, and AdB] for all participating hospitals. The biased-coin principle was used, with a threshold of two. As patients could differ between the participating hospitals in terms of diagnosis and demographic factors (e.g. age), and because these factors are important prognostic factors for RTW [47], randomization was stratified for gender, age (age groups: 18-54 and 55-63 years), and hospital, so as to prevent bias due to unequal randomization. Patients were numbered with three-digit sequential Patient Identification Numbers (PINs). Minimization was applied to equalize group sizes. These PINs were used on the patient questionnaires, which were collected by the research team [AZ, SvH, FD, LJ or AdB].

\section{Blinding}

Patients, healthcare professionals, and researchers were not blinded for the group assignment. One of the researchers [AZ, SvH, FD, or LJ] contacted the healthcare professional and the patient after randomization, so that the first meeting could be scheduled (when patient was randomized to the intervention group). The unique PIN was used for blind analysis of the data by the researcher.

\section{Statistical Analysis}

Data derived from the patient questionnaires was verified by means of a $20 \%$ double check. The primary outcome, RTW, was $100 \%$ double checked [AZ, AdB]. All patients were included in the analysis in accordance with the intention-totreat (ITT) principle. However, patients who died during the study or follow-up period were excluded from the survival analysis. All analyses were performed using the statistical package IBM SPSS Statistics 25.

Possible differences between the intervention and control groups at baseline were verified using Student's t-test for continuous variables and the $\chi^{2}$ test for categorical data. A p-value of $\leq 0.05$ was considered as statistically significant. The primary outcome, days until RTW (either fulltime or partial), was analyzed using the Kaplan-Meier survival method and differences between the intervention and control groups were analyzed using the log-rank test. When the RTW event did not happen, i.e. the patient was no longer 'at risk' of RTW (no further data was available), then these patients were censored. A sensitivity analysis was performed excluding those who stopped participating in the study or were not reported as being on sick leave at any measurement point during the entire study period. After that, a Cox regression analysis was used to represent the estimated hazard ratio (HR) with $95 \%$ confidence interval for the time until RTW. The dependent variable was RTW at twelve months after baseline and the independent variable was group of randomization.

If there was a significant difference in the prognostic factors between the groups at baseline, then the primary outcome variable was adjusted for these factors in a multivariate Cox- regression analysis.

The relative risk (RR) with $95 \%$ confidence interval was presented for employment status at twelve months after baseline, comparing the intervention group with the control group.

Linear mixed models (LMMs) were applied to the longitudinal data to examine differences over time between the intervention and the control group in respect of the secondary outcomes (quality of life, work ability, and work limitations). In the LMM model, the baseline and three, six, nine, and twelve-month scores were included as dependent variables for quality of life and work ability. In the case of work limitations, scores at baseline were not available because patients had to be working then, so only three, six, nine, and twelve-month work-limitation scores were included in the LMM model. We included the fixed effects randomization group and time, and the interaction effect randomization group*time. The subjects were included as random effects. The interaction term (randomization group*time) indicated a difference in time on the outcome (quality of life, work ability, and work limitations) between the study groups. The effects of the study groups could be interpreted as a difference between the groups (control versus intervention) over the follow-up period. A p-value of $\leq 0.05$ was considered as statistically significant. If there was a statistically significant interaction (randomization group* time) effect, then a T-test was applied per time measurement.

\section{Results}

\section{Recruitment and Participant Flow}

Patients were enrolled in the GIRONA study between May 2015 and May 2017, the original period of recruitment May 2015-July 2016 was extend to May 2017 due to the slow inclusion rate. The follow-up period lasted twelve months. Figure 1 presents the participation flow. In total, 88 patients were included; of these, 46 were randomized to the control group and 42 to the intervention group. Accordingly, 


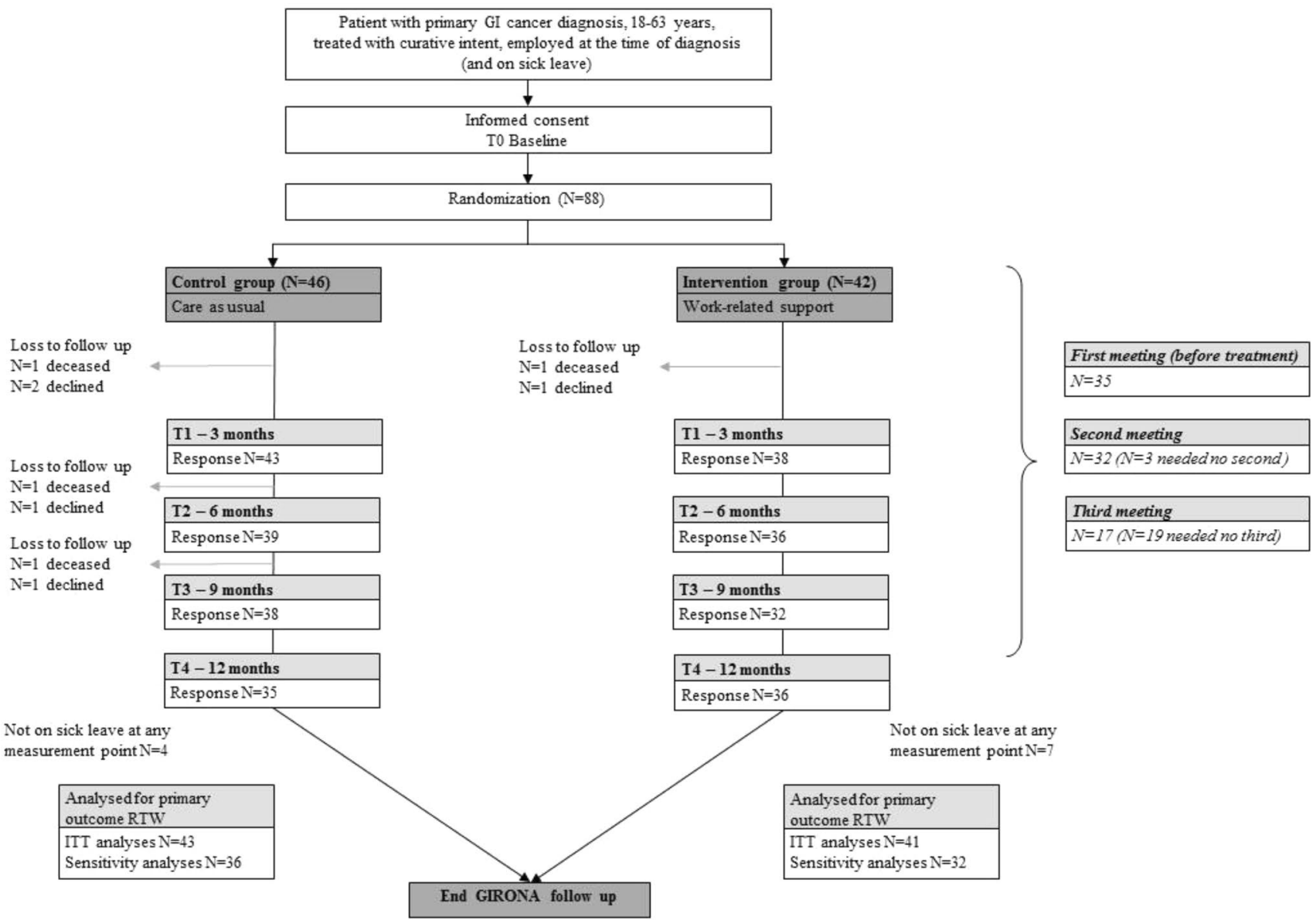

Fig. 1 Participant flow diagram

the baseline questionnaire was filled out by 88 participants (100\%). The response rates for the remaining questionnaires were: $\mathrm{T} 1$ at three months, $\mathrm{N}=81(92 \%)$; $\mathrm{T} 2$ at 6 months, $\mathrm{N}=75$ (85\%); T3 at nine months, $\mathrm{N}=70$ (80\%); and $\mathrm{T} 4$ at twelve months, $\mathrm{N}=71$ (81\%). At baseline, 64 patients were already on sick leave. Nine more reported having taken sick leave at T1 or T2, and eleven were not on sick leave at any measurement point during the study period.

Reasons for not returning one of more questionnaires included death $(\mathrm{N}=4$; three in the control group and one in the intervention group), withdrawal from the study $(\mathrm{N}=5)$ or unknown despite reminders $(\mathrm{N}=21)$.

\section{Patients' Characteristics at Baseline}

Table 1 summarizes the baseline participant characteristics for the intervention and control groups. Of the 88 patients, $67 \%$ were male $(\mathrm{N}=58)$ with an average age of 55 years (SD 7.2 years). The most common type of diagnosis was colon cancer $(\mathrm{N}=58 ; 66 \%)$, followed by rectal cancer $(\mathrm{N}=18$; $21 \%$ ). There were no statistically significant differences at baseline between the intervention and control groups in any sociodemographic, clinical, work-related or health-related characteristic.

Treatments are presented at T1 3 month because patients were included at the moment of diagnosis and therefore not available at T0. At T1 80 patients filled out the question about the treatment they received so far; one did not had any treatment yet, 39 patients underwent surgery, two patients received chemotherapy, one patient had radiotherapy as treatment and 37 patients filled out that they received a combination of operation- chemo and radiotherapy.

\section{The Tailored Work-Related Support Intervention}

Of the 42 patients who were randomized to the intervention group, two declined to take part in the intervention meetings before the first was planned, one died before the first could be planned, and one declined after a meeting was planned. Of the remaining 38 patients in the intervention group, 20 (53\%) were referred to support type A (oncological nurse) and 18 (47\%) to type B (OOP). None was referred to type C 
Table 1 Patients' baseline characteristics

\begin{tabular}{|c|c|c|c|c|c|}
\hline \multirow{2}{*}{$\begin{array}{l}\text { Patient characteristics } \\
\text { Sociodemographic }\end{array}$} & \multicolumn{2}{|c|}{$\begin{array}{l}\text { Intervention group } \\
\mathrm{N}=42^{*}\end{array}$} & \multicolumn{2}{|c|}{$\begin{array}{l}\text { Control group } \\
\mathrm{N}=46^{*}\end{array}$} & \multirow[t]{2}{*}{ p value ${ }^{* * *}$} \\
\hline & & & & & \\
\hline Age (years) & 54 & \pm 7.7 & 56 & \pm 6.6 & 0.26 \\
\hline Gender ( $\%$ male $)$ & $64 \%$ & & $67 \%$ & & 0.76 \\
\hline \multicolumn{6}{|l|}{ Marital status } \\
\hline Married/cohabiting & 33 & & 39 & & \multirow[t]{3}{*}{0.27} \\
\hline Single & 8 & & 4 & & \\
\hline Divorced/widowed & 1 & & 3 & & \\
\hline \multicolumn{6}{|l|}{ Main wage earner } \\
\hline Yes & 19 & & 21 & & \multirow[t]{3}{*}{0.90} \\
\hline No, my partner is & 3 & & 4 & & \\
\hline Equal with partner & 18 & & 17 & & \\
\hline \multicolumn{6}{|l|}{ Gross monthly income } \\
\hline$\leq € 1000$ & 1 & & 2 & & \multirow[t]{5}{*}{0.89} \\
\hline$€ 1001-€ 2000$ & 9 & & 11 & & \\
\hline$€ 2001-€ 3000$ & 15 & & 20 & & \\
\hline$€ 3001-€ 4000$ & 6 & & 4 & & \\
\hline$\geq € 4001$ & 1 & & 1 & & \\
\hline \multicolumn{6}{|l|}{ Educational attainment } \\
\hline Low & 9 & & 15 & & \multirow[t]{3}{*}{0.46} \\
\hline Intermediate & 17 & & 15 & & \\
\hline High & 16 & & 15 & & \\
\hline \multicolumn{6}{|l|}{ Clinical characteristics } \\
\hline \multicolumn{6}{|l|}{ Cancer diagnosis } \\
\hline Stomach & - & & 1 & & \multirow[t]{8}{*}{0.61} \\
\hline Liver & 1 & & 1 & & \\
\hline Gallbladder & - & & 1 & & \\
\hline Small intestine & 1 & & - & & \\
\hline Colon & 30 & & 28 & & \\
\hline Rectal & 7 & & 11 & & \\
\hline Pancreatic & 2 & & 4 & & \\
\hline Anal & 1 & & - & & \\
\hline \multicolumn{6}{|l|}{ Treatment (T1 3 months) $* * *$} \\
\hline None & 0 & & 1 & & \multirow[t]{5}{*}{0.32} \\
\hline Surgery $(\mathrm{S})$ & 18 & & 21 & & \\
\hline Chemotherapy (CT) & 2 & & 0 & & \\
\hline Radiotherapy (RT) & 1 & & 0 & & \\
\hline Combination (S-CT-RT) & 21 & & 16 & & \\
\hline \multicolumn{6}{|l|}{ Work-related characteristics } \\
\hline \multicolumn{6}{|l|}{ Occupational sector } \\
\hline Healthcare/education & 11 & & 5 & & \multirow[t]{6}{*}{0.08} \\
\hline Administrative & - & & 6 & & \\
\hline Sales & 3 & & 1 & & \\
\hline Industry/transport/logistics & 8 & & 12 & & \\
\hline Business services & 13 & & 14 & & \\
\hline Other & 7 & & 8 & & \\
\hline Years in current function & 16.4 & \pm 11.5 & 17.35 & \pm 11 & 0.70 \\
\hline Years in paid work & 31.3 & \pm 11 & 32.8 & \pm 9.9 & 0.50 \\
\hline \multicolumn{6}{|l|}{ Employment status } \\
\hline Permanent & 30 & & 41 & & 0.19 \\
\hline Temporary & 3 & & 1 & & \\
\hline
\end{tabular}


Table 1 (continued)

\begin{tabular}{|c|c|c|c|c|c|}
\hline \multirow{2}{*}{$\begin{array}{l}\text { Patient characteristics } \\
\text { Self-employed }\end{array}$} & \multicolumn{2}{|c|}{$\begin{array}{l}\text { Intervention group } \\
\mathrm{N}=42^{*}\end{array}$} & \multicolumn{2}{|c|}{$\begin{array}{l}\text { Control group } \\
\mathrm{N}=46^{*}\end{array}$} & \multirow[t]{2}{*}{$\mathrm{p}$ value ${ }^{* * *}$} \\
\hline & 6 & & 3 & & \\
\hline Hours under current contract (per week) & 37 & 10.8 & 35 & 9.3 & 0.49 \\
\hline \multicolumn{6}{|l|}{ Physical workload $* * * *$} \\
\hline VBBA Score 0-28 & 4.3 & \pm 5.1 & 4.9 & \pm 4.6 & 0.56 \\
\hline \multicolumn{6}{|l|}{ Importance of work $* * * *$} \\
\hline Score 0-100 & 51.6 & \pm 29.5 & 47.7 & \pm 29.2 & 0.53 \\
\hline \multicolumn{6}{|l|}{ Reconsider importance of work } \\
\hline No & 30 & $71 \%$ & 27 & $60 \%$ & 0.26 \\
\hline \multicolumn{6}{|l|}{ Support from family and friends } \\
\hline No, no need of & 16 & & 17 & & 0.56 \\
\hline No, but need it & 1 & & - & & \\
\hline Yes, I have this support & 25 & & 29 & & \\
\hline \multicolumn{6}{|l|}{ Support from work environment } \\
\hline No, no need of & 4 & & 10 & & 0.15 \\
\hline No, but need it & 2 & & 5 & & \\
\hline Yes, I have this support & 35 & & 31 & & \\
\hline \multicolumn{6}{|l|}{ Health-related characteristics } \\
\hline \multicolumn{6}{|l|}{ Fatigue (general) $* * * *$} \\
\hline MFI Score 0-20 & 12 & \pm 5.1 & 11.9 & \pm 5.1 & 0.91 \\
\hline \multicolumn{6}{|l|}{ Depression**** } \\
\hline CESD Score 0-60 & 12.1 & \pm 9.9 & 11.4 & \pm 9.1 & 0.74 \\
\hline \multicolumn{6}{|l|}{ Cognitive functioning $* * * *$} \\
\hline CSCW-DW Score 0-100 & 21 & \pm 15.5 & 27.1 & \pm 18.8 & 0.1 \\
\hline
\end{tabular}

* Due to missing values or rounding differences, numbers may approach the total $\mathrm{N}$ and $100 \%$

${ }^{* *}$ Continuous variables mean \pm standard deviation 1 Nominal and ordinal variables $(\mathrm{N})$ with percentages. Student's $t$ test for continuous variables and $\chi^{2}$ test for ordinal and nominal variables

*** Treatments are presented at T1 ( 3 month) because patients were included at the moment of diagnosis and therefore not available at $\mathrm{T} 0$

***** The higher the score, the higher the level of physical workload, importance of work, fatigue, feelings of depression, and cognitive functioning problems

(multidisciplinary team). Further details of the intervention procedure outcomes are described in the process evaluation article (submitted for publication). No adverse events were reported, either by patients or by healthcare professionals.

\section{Primary outcome: time until RTW}

\section{Intention-to-Treat Analysis (ITT)}

The median time from sick leave until fulltime or partial RTW was 233 days (187-279 days, 95\% CI) for the control group and 190 days (139-240 days, 95\% CI) for the intervention group. There was no statistically significant difference between these groups concerning time from sick leave until fulltime or partial RTW (log-rank $\mathrm{p}$ value $=0.37$ ). Figure 2a shows the Kaplan-Meier survival analysis; the vertical line represents the 11 patients who were not on sickleave at any time during the study, reflecting their period until RTW as 0 days (since the event 'RTW from sick leave' did not happen). The hazard ratio (HR) for RTW (either fulltime or partial) at twelve months after baseline was 1.2 (95\% CI 0.77-2.0) for the intervention group versus the control group.

\section{Sensitivity Analysis}

Figure $2 \mathrm{~b}$ shows the subgroup Kaplan-Meier survival analysis, excluding patients who did were not on sick leave at any point between baseline (T0) and twelve months (T4) $(\mathrm{N}=11)$ and those who stopped participating $(\mathrm{N}=5)$. Patients who were randomized to the intervention group but declined to take part in the intervention received control-group questionnaires and were analyzed as control-group participants $(\mathrm{N}=2)$. Those who died $(\mathrm{N}=4)$ during the study were again excluded. The median time from sick leave until fulltime 

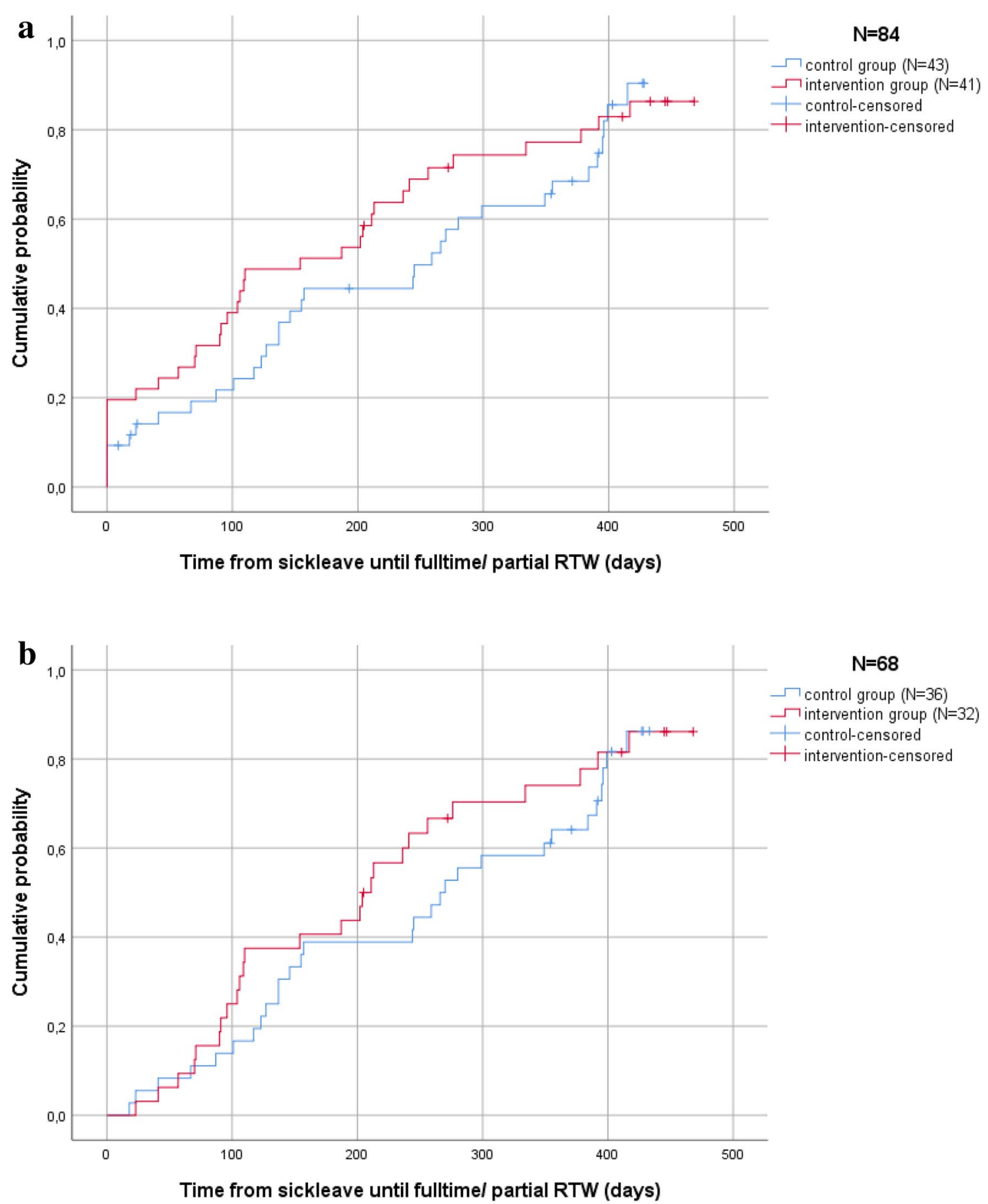

Fig. 2 a Kaplan Meier (KM) survival ITT analysis for time until return to work (fulltime or partial).b Kaplan Meier (KM) survival Sensitivity analysis for time until return to work (fulltime or partial)

or partial RTW was 260 days (214-305 days, 95\% CI) for the control group and 228 days (177-278 days, 95\% CI) for the intervention group. This was not statistically significant (log-rank p value $=0.39$ ).

\section{Secondary Outcomes}

\section{Relative Risk (RR): Returning to Work}

The RTW (fulltime or partial) rate at twelve months follow-up, was calculated using the available data from 70 
patients (intervention group $\mathrm{N}=36$, control group $\mathrm{N}=34$ ), was $83.3 \%$ for the intervention group and $73.5 \%$ for the control group $(p=0.32)$. The RR of returning to work at twelve months follow-up after baseline was 1.13 (95\% CI $0.88-1.45)$ for the intervention group versus the control group.

\section{Effects Over Time for Quality of Life, Work Ability, and Work Limitations}

Table 2 presents the outcomes over time of quality of life, work ability, and work limitations. All of these secondary outcomes improved to a statistically significant extent over time. However, the main effects per group (intervention versus control) were not statistically significant for either component—physical score (PCS) and mental score (MCS) —of the quality of life outcomes, or for work ability and work limitations (respectively, PCS $\mathrm{p}=0.09 / \mathrm{MCS} p=0.45$, work ability $p=0.14$, and work limitations $p=0.33$ ). The interaction effects of randomization group*time were not statistically significant (randomization group*time; PCS $p=0.59$ / MCS $p=0.13$ and work ability $p=0.15$ ). For both groups, the PCS had its lowest score at three months from baseline and had increased again at nine months from baseline.

For the work limitations outcomes, the intervention group scored fewer limitations at both three and six months after baseline than the control group. The main effect over time was statistically significant $(\mathrm{p}=0.01)$. On two (mental-interpersonal demands and output demands) of the four subscales of the work-limitations questionnaire, there was a statistically significant effect on the main interaction effect of randomization group*time (respectively, $\mathrm{p}=0.02$ and $\mathrm{p}=0.01$ ). This effect was statistically significant at measurement $\mathrm{T} 1$ after three months (WLQ general, $\mathrm{p}=0.05$; mental-interpersonal demands $p=0.03$ and output demands $p=0.04$ ).

\section{Discussion}

The hypothesis of this study was that offering tailored workrelated support early in the clinical diagnostic phase would lead to enhancement of RTW and therefore result in fewer days of sick leave. There was no statistically significant decrease in RTW, although numerically the interventiongroup patients returned to work after a mean of 190 days of sick leave which a difference of 43 days compared with the control-group average.

The RTW rates at twelve months after baseline were relatively high for both groups. But again, neither this result nor the other secondary outcomes quality of life and work ability (outcomes between the groups over time) were statistically significant. Work limitations did show a significant result, in the sense that the intervention group had fewer work limitations at three months after baseline.

\section{Methodological Consideration}

The major methodological concern, and the explanation for the lack of statistical significant differences between the groups, is the power of the study. Unfortunately, we were unable to include the number of patients prescribed under the predetermined sample-size calculation (309intotal). This was possibly due to other ongoing (interfering) studies, or to the timing of the study's introduction to prospective participants (in the diagnostic phase). Based on previous studies, the RTW rate with 'care as usual' was $63 \%$, compared with $81 \%$ for the group who received a work-related support intervention. We did reach those rates in this study, too (RTW at twelve months after baseline: control $73.5 \%$ versus $82.3 \%$ for the intervention group), but without statistical significance due to its insufficient power.

\section{Interpretation of the Findings}

Despite the lack of statistically significant results, this study nevertheless produced some potentially promising outcomes. First, the number of days between taking sick leave and RTW was lower for those who received the workrelated support intervention. These results are encouraging, as they were found at three to six months after baseline (see Fig. 2 and Table 2)-i.e., during the period in which the intervention was being performed. From the results of the process evaluation (submitted for publication), we know that the first meeting before treatment was difficult to conduct in practice, but these meetings were held as soon as possible and the second meetings were mostly performed within six months of the first.

Another promising finding of our study is that the RTW rates at twelve months after baseline are relatively high for both groups: $83.3 \%$ for the intervention group, compared with $73.5 \%$ for the control group.

The results of the systematic review conducted by Mehnert [48] underline these high RTW rates. That review reported a mean of $63 \%$ (range $50-81 \%$ ) of participants managing to RTW or to stay in work during treatment at twelve months following the diagnosis of cancer. Our intervention was developed to support and inform patients about work, RTW, and related problems at an early stage. So although we expected the RTW rate of the control group to be lower than that of the intervention group, because they did not receive work-related support, its result is still a positive finding of this study. Despite the lack of work-related support, their RTW percentage showed to be relatively high by comparison with RTW percentage published in the review of Mehnert [48]. One possible explanation for this is 


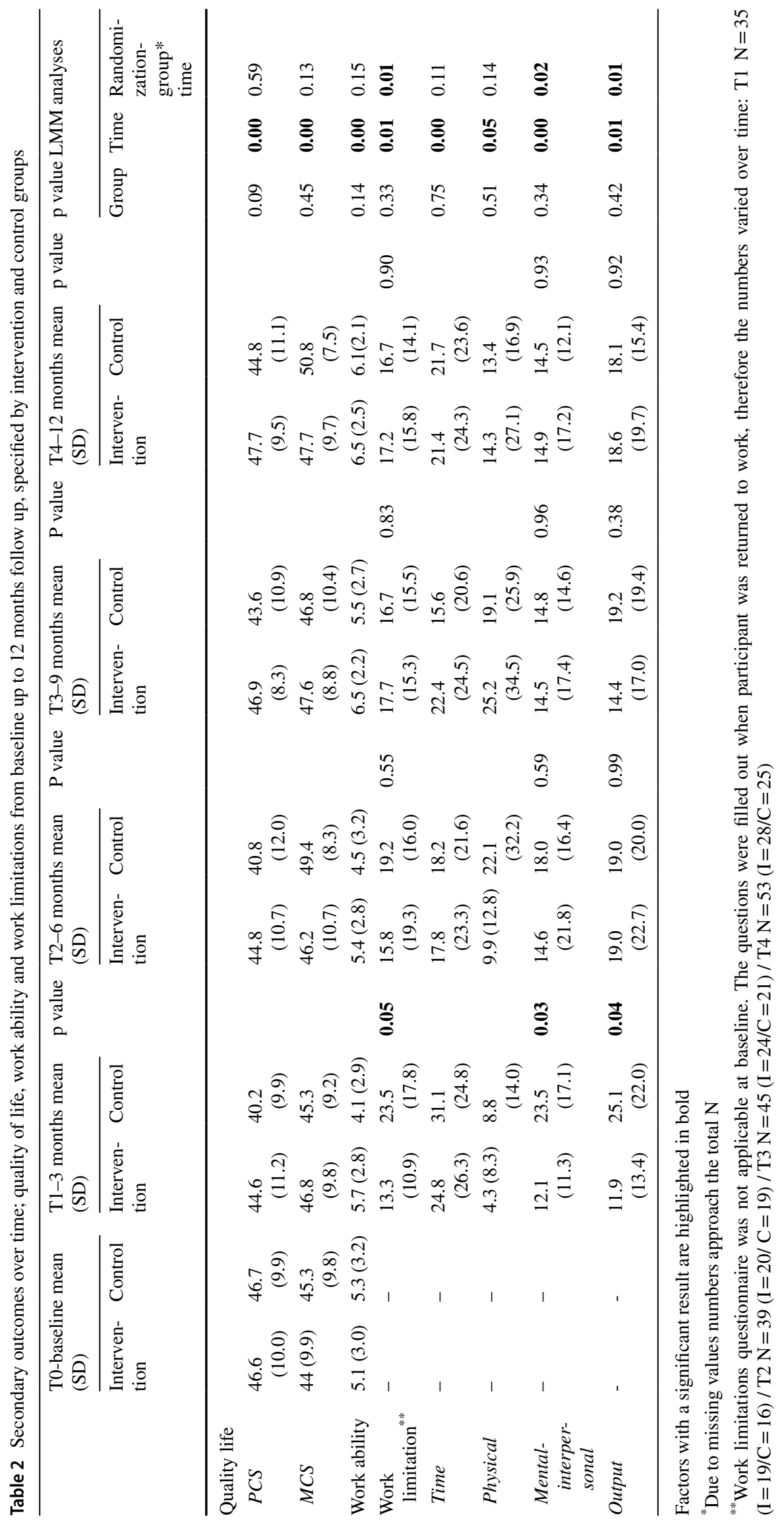


raised awareness of the importance of work even among the control group in our study, because their mere inclusion in the study drew their attention to possible RTW problemsfirstly because 'work' attracted more overall social interest during the period of the study. Secondly because all participants were informed about its aim before they signed their informed consent to participate, which could have resulted in 'information-biased' results. Moreover, the questionnaires sent to both groups, control and intervention, included workrelated items. Patients were therefore constantly reminded about the importance of work. As a consequence, it could be that control-group patients themselves took action to seek the support they needed. As outlined earlier (Table 3), for example, control-group patients had paid twice as many visits to an occupational physician than participants in the intervention group at three months after baseline. So even though this may also explain the lack of statistically significant differences between the groups, we may assume that informing patients at an early stage triggered them to think about RTW. This further stresses the importance of including work-related information in day-to-day clinical practice.

Alongside the rather broad range of RTW rates described in the systematic review conducted by Mehnert [48], a few other studies have also reported on the length of sick leave (i.e. days until RTW). In this respect, too, our study reveals considerable differences. Some patients did not report sick at all, whereas others had still not returned to work twelve months after baseline. However, the systematic review of Mehnert [48] was based on 64 studies and so many different characteristics were included. A mean duration of 151 days of sick leave was reported [48]. Considerable differences in this outcome are also described in the other literature; for example, one study reported a mean of 349 days [49] and another 86 days [50]. These differences are related to a number of factors, such as cancer site, treatment type, physical complaints like level of fatigue, and the level of workload [44]. Because of this, it is complicated to compare the RTW rates and/or days until RTW of patients diagnosed with cancer. In other words, these differences are of importance to the outcome and differ per individual. Although some factors are non-modifiable, it is important to identify those patients 'at risk', as also determined by Kiasuwa et al. [51]. All of which further underlines the fact that an intervention must be tailored to the needs of the patient concerned. A 'one-size-fits-all' intervention inevitably disregards these different factors. To identify those patients at risk and to tailor the support, we developed a decision diagram with three kind of supports; support A (mild work related problems), B (severe work-related problems) and C (complex work-related problems), respectively. From the results of our study, no patients needed the support of a multidisciplinary team (support C). However, we could not conclude that this support is therefore not needed.

Still, we think that this option must be available, as this was discussed in the development of the intervention with an expert panel [28]. In line with this the clinical relevance of a multi-disciplinary approach [52] and the opinions of the expert panel support $\mathrm{C}$ is of value.

The intervention in practice was scored with different key components; recruitment, context, reach, dose delivered, dose received and fidelity. Although the study protocol for the intervention in practice was easy to follow according

Table 3 Work-related support other than the GIRONA tailored intervention, specified by intervention and control groups

\begin{tabular}{|c|c|c|c|c|c|c|c|c|}
\hline & \multicolumn{2}{|l|}{ T1-3 months } & \multicolumn{2}{|l|}{ T2-6 months } & \multicolumn{2}{|l|}{ T3-9 months } & \multicolumn{2}{|c|}{ T4-12 months } \\
\hline & Intervention & Control & Intervention & Control & Intervention & Control & Intervention & Control \\
\hline \multicolumn{9}{|c|}{ Work-related support outside the hospital ${ }^{*}$} \\
\hline Reintegration agency & 1 & - & - & - & - & - & - & - \\
\hline Reintegration coach & 1 & 2 & 1 & 1 & - & 1 & - & - \\
\hline Rehabilitation program & 3 & 1 & 1 & - & 4 & 4 & 3 & 1 \\
\hline Other ${ }^{* * *}$ & 6 & 2 & 1 & 6 & 3 & 5 & 4 & 4 \\
\hline \multicolumn{9}{|c|}{ Other healthcare professionals ${ }^{* *}$} \\
\hline Occupational physician & 12 & 24 & 13 & 17 & 10 & 18 & 10 & 13 \\
\hline Social worker & 2 & 2 & - & 2 & - & 1 & 2 & 2 \\
\hline Other $^{* * * * *}$ & 5 & 3 & 2 & 6 & 4 & 7 & 5 & 5 \\
\hline
\end{tabular}

*Question: "Have you received support outside the hospital for work-related problems?"

**Question: "Have you visited other healthcare professionals for support with work-related problems in the past three months?"

***Intervention group, e.g. occupational physician, employer, social security agency (UWV), general practitioner, cancer care consultant, psychologist. Control group, e.g. occupational physician, physiotherapist, psychologist, financial advisor, vitality coach

****Intervention group, e.g. colleagues, general practitioner, oncological occupational physician, psychologist, physiotherapist, social security agency (UWV).Control group, e.g. manager, employer, labor expert, corporate counselor, psychologist (incl. sports psychologist), oncological nurse, general practitioner 
to the healthcare professionals, there are mainly logistical issues that needs to be revised. Particularly the duration and timing of the intervention resulted in a low fidelity score (protocol adherence) for the oncological occupational physician, while the oncological nurses scored better in the total fidelity score. One of the limitations of the intervention in practice which must be taken into account is that the intervention patients were spread over 16 hospitals which might have caused heterogeneity The inclusion of 16 hospitals resulted in nurses in each hospital not having many work-related support meetings. It is possible that meetings therefore were not completely conducted as intended. The majority of the patients were satisfied and found the intervention useful and the healthcare professionals acknowledge the importance of the awareness of work- related support with the clinical setting.

\section{Strengths and Limitations}

One strength of our intervention study is that we provided 'tailored' work-related support, which is an innovative aspect within psychosocial work-related support. At the present time there are more studies about RTW interventions; however, the intervention we developed was also innovative in that it supported patients with work-related problems from an early stage of their diagnosis. Supporting patients with work-related issues in the clinical setting is a subject acknowledged as important by the participating healthcare professionals in this study, although it is still rarely discussed in such an early phase $[17,18]$.

Another strength of the intervention was the involvement of different healthcare professionals to ensure tailored workrelated support. The oncological nurse is the first professional in a position to discuss work with the patient, to notice possible (experienced) work-related problems, and possibly also to involve the second important healthcare professional: the oncological occupational physician (OOP), who possesses specialized knowledge about cancer in relation to work [29]. The OOP is involved in the clinical setting instead of being employed by an occupational health service, which makes them even more independent to patients' perception.

On the other hand, one major limitation of this studyas already mentioned-was its inability to include a sufficient number of patients. Another was the differences in the treatment of the different GI diagnoses, because these could influence RTW. At baseline, however, the intervention and control groups were equal in respect of their diagnoses.

\section{Study Design Considerations}

This study was performed as a randomized controlled trial (RCT), a research method whereby participants are assigned randomly to a group so as to preclude differences between groups. Nevertheless, in this case two factors might have caused selection bias. First, healthcare professionals may not have informed some eligible patients about the study, thus resulting in the number of participating patients being rather low. If this was the case, one possible explanation is that patients nowadays are often asked to participate in more than one study; however, they should not be overloaded with such requests, especially when they have just been diagnosed with cancer. Petersen et al. [18] point out that occupational support is not yet integrated with clinical care and so, despite interest in this kind of work-related support, healthcare providers could be critical towards patient recruitment. Unfortunately, we have no data concerning all the eligible patients because this was not recorded efficiently at the participating hospitals. Moreover, those who are included in the study are likely to be ones willing to participate because they know the value of research and are interested in the subject.

\section{Recommendations for Research and Practice}

Work-related support is an issue which is becoming increasingly recognized in day-to-day clinical and psycho-oncological practice. However, it is recommended that research continue in order to develop a consistent foundation and better knowledge whereby healthcare professionals can support patients (in an early phase) with work-related issues $[18,53,54]$. Another recommendation for future research, when the intervention is proven effective, is to perform an economic evaluation from a societal perspective including costs of intervention, loss of productivity and costs of sick leave days. Although no statistically significant differences were found in this study, the results are still interesting: a difference of 43 days less sick leave for the intervention group, compared with the 'care as usual' control group, plus the positive experiences reported by both patients and healthcare professionals. The value of an economic evaluation is important showing that tailored work-related support is beneficial for the individual patient as well as for society at large will provide more knowledge and generate greater urgency to implement such support in day-to-day practice.

\section{Generalizability}

Because we used a multicenter RCT design and included 16 different hospitals distributed over the Netherlands, we can interpret the results of this study as applicable to the Netherlands as a whole. Individuals who become ill are protected by the social security system in the Netherlands. This Act is called the 'Improved Gatekeepers Act', is active for the first two years of sick leave and partly covers the wage loss. Both employee and employer need are obliged to actively insert effort for the return to work process. The occupational 
physician also takes part in this process as well. Generalizability to other countries is possible if their clinical healthcare provision is comparable with the situation described in this study. It should be noted, however, that we used OOPs in our study: physicians specialized in occupational support for oncology-related issues [29]—a specific type of healthcare professional that may not available everywhere, or whose role and remit could be different in other countries.

\section{Conclusion}

Our analysis shows a possible positive effect of the intervention on enhancement of RTW. The results add novel findings about early, tailored work-related support in the clinical setting.

Acknowledgements We would like to thank all participants for taking part in the GIRONA study and sharing their information with regard to the intervention, and the participating hospitals and healthcare professionals for their participation. We also would like to thank C. Oostveen (OOP) and L. de Bruijn (OOP) for their participation.

Author Contributions $\mathrm{AZ}, \mathrm{SvH}, \mathrm{FD}$ and $\mathrm{LJ}$ were responsible for data collection. AZ and AdB were responsible for the data analyses. AZ was responsible for the draft manuscript. All the authors have read and corrected draft versions of the manuscript and approved the final version.

Funding This study was funded by a Grant from the Dutch Cancer Society (UVA2012-5619).

\section{Compliance with Ethical Standards}

Conflict of interest The authors declare that they have no conflict of interest.

Ethical Approval All procedures performed in studies involving human participants were in accordance with the ethical standards of the institutional and/or national research committee and with the 1964 Helsinki declaration and its later amendments or comparable ethical standards.

Informed Consent Informed consent was obtained from all individual participants included in the study.

Open Access This article is licensed under a Creative Commons Attribution 4.0 International License, which permits use, sharing, adaptation, distribution and reproduction in any medium or format, as long as you give appropriate credit to the original author(s) and the source, provide a link to the Creative Commons licence, and indicate if changes were made. The images or other third party material in this article are included in the article's Creative Commons licence, unless indicated otherwise in a credit line to the material. If material is not included in the article's Creative Commons licence and your intended use is not permitted by statutory regulation or exceeds the permitted use, you will need to obtain permission directly from the copyright holder. To view a copy of this licence, visit http://creativecommons.org/licenses/by/4.0/.

\section{References}

1. Naughton MJ, Weaver KE. Physical and mental health among cancer survivors: considerations for long-term care and quality of life. N C Med J. 2014;75(4):283-6.

2. Berger AM, Mooney K, Alvarez-Perez A, Breitbart WS, Carpenter KM, Cella D, et al. Cancer-related fatigue, version 22015. J Natl Compr Cancer Netw. 2015;13(8):1012-39.

3. Harrington CB, Hansen JA, Moskowitz M, Todd BL, Feuerstein M. It's not over when it's over: long-term symptoms in cancer survivors: a systematic review. Int J Psychiatry Med. 2010;40(2):163-81.

4. Weis J. Cancer-related fatigue: prevalence, assessment and treatment strategies. Expert Rev. Pharmacoecon. Outcomes Res. 2011;11(4):441-6.

5. Duijts SF, van Egmond MP, Spelten E, van Muijen P, Anema JR, van der Beek AJ. Physical and psychosocial problems in cancer survivors beyond return to work: a systematic review. Psychooncology. 2014;23(5):481-92.

6. Wells M, Williams B, Firnigl D, Lang H, Coyle J, Kroll T, et al. Supporting 'work-related goals' rather than 'return to work' after cancer? A systematic review and meta-synthesis of 25 qualitative studies. Psychooncology. 2013;22(6):1208-19.

7. Amir Z, Neary D, Luker K. Cancer survivors' views of work 3 years post diagnosis: a UK perspective. Eur. J. Oncol. Nurs. 2008;12(3):190-7.

8. Duijts SFA, van Egmond MP, Gits M, van der Beek AJ, Bleiker EM. Cancer survivors' perspectives and experiences regarding behavioral determinants of return to work and continuation of work. Disabil Rehabil. 2016;39:1-9.

9. Rasmussen DM, Elverdam B. The meaning of work and working life after cancer: an interview study. Psychooncology. 2008;17(12):1232-8.

10. Peteet JR. Cancer and the meaning of work. Gen Hosp Psychiatry. 2000;22(3):200-5.

11. Kale HP, Carroll NV. Self-reported financial burden of cancer care and its effect on physical and mental health-related quality of life among US cancer survivors. Cancer. 2016;122(8):283-9.

12. de Boer AG, Bruinvels DJ, Tytgat KM, Schoorlemmer A, Klinkenbij1 JH, Frings-Dresen MH. Employment status and work-related problems of gastrointestinal cancer patients at diagnosis: a cross-sectional study. BMJ Open. 2011;1(2): e000190.

13. Bains M, Munir F, Yarker J, Steward W, Thomas A. Return-towork guidance and support for colorectal cancer patients: a feasibility study. Cancer Nurs. 2011;34(6):E1-.

14. Tamminga SJ, de Boer AG, Bos MM, Fons G, Kitzen JJ, Plaisier $\mathrm{PW}$, et al. A hospital-based work support intervention to enhance the return to work of cancer patients: a process evaluation. J Occup Rehabil. 2012;22(4):565-78.

15. Henderson M, Glozier N, Holland EK. Long term sickness absence. BMJ. 2005;330(7495):802-3.

16. Dekkers-Sanchez PM, Hoving JL, Sluiter JK, Frings-Dresen MH. Factors associated with long-term sick leave in sick-listed employees: a systematic review. Occup Environ Med. 2008;65(3):153-7.

17. Pryce J, Munir F, Haslam C. Cancer survivorship and work: symptoms, supervisor response, co-worker disclosure and work adjustment. J Occup Rehabil. 2007;17(1):83-92.

18. Petersen KS, Momsen AH, Stapelfeldt CM, Olsen PR, Nielsen CV. Return-to-work intervention during cancer treatment: the providers' experiences. Eur J Cancer Care. 2018;27(2):e12793.

19. Boyajian RN, Grose A, Grenon N, Roper K, Sommer K, Walsh M, et al. Desired elements and timing of cancer survivorship care: one approach may not fit all. J Oncol. Pract. 2014;10(5):e293-e298298. 
20. Zaman AG, Tytgat KM, Klinkenbijl JH, Frings-Dresen MH, de Boer AG. Design of a multicentre randomized controlled trial to evaluate the effectiveness of a tailored clinical support intervention to enhance return to work for gastrointestinal cancer patients. BMC Cancer. 2016;16:303.

21. Leensen MCJ, Groeneveld IF, Rejda T, Groenenboom P, van Berkel S, Brandon T, et al. Feasibility of a multidisciplinary intervention to help cancer patients return to work. Eur. J. Cancer Care. 2018;27(2):e12690.

22. Stone DS, Ganz PA, Pavlish C, Robbins WA. Young adult cancer survivors and work: a systematic review. J. Cancer Surviv. 2017;11(6):765-81.

23. Groeneveld IF, de Boer AG, Frings-Dresen MH. A multidisciplinary intervention to facilitate return to work in cancer patients: intervention protocol and design of a feasibility study. BMJ Open. 2012;2(4):e001321.

24. Stapelfeldt CM, Labriola M, Jensen AB, Andersen NT, Momsen AM, Nielsen CV. Municipal return to work management in cancer survivors undergoing cancer treatment: a protocol on a controlled intervention study. BMC Public Health. 2015;15:720.

25. Tamminga SJ, de Boer AG, Verbeek JH, Taskila T, Frings-Dresen $\mathrm{MH}$. Enhancing return-to-work in cancer patients, development of an intervention and design of a randomised controlled trial. BMC Cancer. 2010;10:345.

26. Moher D, Hopewell S, Schulz KF, Montori V, Gotzsche PC, Devereaux PJ, et al. CONSORT 2010 explanation and elaboration: updated guidelines for reporting parallel group randomised trials. Int J Surg (Lond. Engl.). 2012;10(1):28-55.

27. Schulz KF, Altman DG, Moher D. CONSORT 2010 statement: updated guidelines for reporting parallel group randomised trials. BMJ (Clini Res Ed.). 2010;340:c332.

28. Zaman A, Tytgat K, van Hezel S, Klinkenbijl JHG, de Boer A, Frings-Dresen MHW. Development of a tailored work-related support intervention for gastrointestinal cancer patients. Eur J Cancer Care. 2018;27(2):e12782.

29. Zaman A, Bruinvels DJ, de Boer A, Frings-Dresen MHW. Supporting cancer patients with work-related problems through an oncological occupational physician: a feasibility study. Eur J Cancer Care. 2017;26(5):e12690.

30. Zaman A, Tytgat K, Klinkenbijl JHG, de Boer A, Frings-Dresen MHW. Process evaluation of a tailored work-related support intervention for patients diagnosed with gastrointestinal cancer. J Cancer Surv. 2020;14(1):59-71.

31. Gandek B, Ware JE, Aaronson NK, Apolone G, Bjorner JB, Brazier JE, et al. Cross-validation of item selection and scoring for the SF-12 Health Survey in nine countries: results from the IQOLA project: International Quality of Life Assessment. J Clin Epidemiol. 1998;51(11):1171-8

32. Ware J Jr, Kosinski M, Keller SD. A 12-item short-form health survey: construction of scales and preliminary tests of reliability and validity. Med Care. 1996;34(3):220-33.

33. Tuomi KIJ, Jahkola A. Work Ability Index, 2nd. revised ed. Helsinki: Finnish Institute of Occupational Health; 1998.

34. de Zwart BC, Frings-Dresen MH, van Duivenbooden JC. Testretest reliability of the Work Ability Index questionnaire. Occupat Med (Oxf Engl.). 2002;52(4):177-81.

35. Lerner D, Amick BC 3rd, Rogers WH, Malspeis S, Bungay K, Cynn D. The Work Limitations Questionnaire. Med Care. 2001;39(1):72-85.

36. Feuerstein M, Hansen JA, Calvio LC, Johnson L, Ronquillo JG. Work productivity in brain tumor survivors. J Occup Environ Med. 2007;49(7):803-11.

37. Tamminga SJ, Verbeek JH, Frings-Dresen MH, De Boer AG. Measurement properties of the Work Limitations Questionnaire were sufficient among cancer survivors. Qual life Res. 2014;23(2):515-25.
38. Smets EM, Garssen B, Bonke B, De Haes JC. The Multidimensional Fatigue Inventory (MFI) psychometric qualities of an instrument to assess fatigue. J Psychosom Res. 1995;39(3):315-25.

39. Rl S. The CES-D scale: A self-report depression scale for research in the general population. Appl Psychol Meas. 1997;1:385-401.

40. Dorland HF, Abma FI, Roelen CA, Smink A, Feuerstein M, Amick $\mathrm{BC}$, et al. The Cognitive Symptom Checklist-Work in cancer patients is related with work functioning, fatigue and depressive symptoms: a validation study. J Cancer Surv. 2016;10(3):545-52.

41. van Veldhoven MMT, Broersen JPJ, Fortuin RJ. Manual Qeustionnaire Perception and Judgment of Work. [In Dutch: Handleiding Vragenlijst Beleving en Beoordeling van de Arbeid (VBBA). Amsterdam 2002.

42. Roelen CA, Koopmans PC, Groothoff JW, van der Klink JJ, Bultmann U. Return to work after cancer diagnosed in 2002, 2005 and 2008. J Occup Rehabil. 2011;21(3):335-41.

43. Nieuwenhuijsen K, Bos-Ransdorp B, Uitterhoeve LL, Sprangers MA, Verbeek JH. Enhanced provider communication and patient education regarding return to work in cancer survivors following curative treatment: a pilot study. J Occup Rehabil. 2006;16(4):647-57.

44. Spelten ER, Verbeek JH, Uitterhoeve AL, Ansink AC, van der Lelie J, de Reijke TM, et al. Cancer, fatigue and the return of patients to work-a prospective cohort study. Eur J Cancer (Oxf Engl.). 2003;39(11):1562-7.

45. de Boer AG, Verbeek JH, Spelten ER, Uitterhoeve AL, Ansink AC, de Reijke TM, et al. Work ability and return-to-work in cancer patients. Br J Cancer. 2008;98(8):1342-7.

46. https://prod.tenalea.net/ctcm/dm/. TENALEA 2009 Assessed Sept 2015.

47. Spelten ER, Sprangers MA, Verbeek JH. Factors reported to influence the return to work of cancer survivors: a literature review. Psychooncology. 2002;11(2):124-31.

48. Mehnert A. Employment and work-related issues in cancer survivors. Crit Rev Oncol. 2011;77(2):109-30.

49. Roelen CA, Koopmans PC, de Graaf JH, Balak F, Groothoff JW. Sickness absence and return to work rates in women with breast cancer. Int Arch Occup Environ Health. 2009;82(4):543-6.

50. Ohguri T, Narai R, Funahashi A, Nishiura C, Yamashita T, Yarita $\mathrm{K}$, et al. Limitations on work and attendance rates after employees with cancer returned to work at a single manufacturing company in Japan. J Occupat Health. 2009;51(3):267-72.

51. Kiasuwa Mbengi R, Otter R, Mortelmans K, Arbyn M, Van Oyen $\mathrm{H}$, Bouland $\mathrm{C}$, et al. Barriers and opportunities for return-to-work of cancer survivors: time for action-rapid review and expert consultation. Syst Rev. 2016;5:35.

52. de Boer AG, Taskila TK, Tamminga SJ, Feuerstein M, FringsDresen $\mathrm{MH}$, Verbeek JH. Interventions to enhance returnto-work for cancer patients. Cochrane Database Syst Rev. 2015;9:Cd007569.

53. Bains M, Yarker J, Amir Z, Wynn P, Munir F. Helping cancer survivors return to work: what providers tell us about the challenges in assisting cancer patients with work questions. J Occup Rehabil. 2012;22(1):71-7.

54. McGrath C, Mihala G, Beesley VL, Lynch BM, Graves N, Gordon LG. "Cancer Put My Life on Hold": work-related challenges among middle-aged adults 12 months after a diagnosis of colorectal cancer. Cancer Nurs. 2017;40(2):160-7.

Publisher's Note Springer Nature remains neutral with regard to jurisdictional claims in published maps and institutional affiliations. 


\section{Affiliations}

\section{A. C. G. N. M. Zaman ${ }^{1}$ (1) K. M. A. J. Tytgat ${ }^{2}$ - J. H. G. Klinkenbij/3,4 F. C. den Boer ${ }^{5}$ - M. A. Brink ${ }^{6}$. J. C. Brinkhuis ${ }^{7}$. D. J. Bruinvels ${ }^{7}$. L. C. M. Dol ${ }^{8}$ - P. van Duijvendijk ${ }^{3}$ - P. H. J. Hemmer ${ }^{9}$ B. Lamme ${ }^{10}$ - O. J. L. Loosveld ${ }^{11}$ - M. M. Mok ${ }^{12}$. T. Rejda ${ }^{13} \cdot$ H. Rutten ${ }^{14} \cdot$ A. Schoorlemmer ${ }^{15}$. D. J. Sonneveld ${ }^{16}$. L. P. S. Stassen ${ }^{17} \cdot$ R. P. Veenstra ${ }^{18} \cdot$ A. van de Ven ${ }^{19}$. $^{2}$ E. R. Velzing ${ }^{20} \cdot$ M. H. W. Frings-Dresen ${ }^{1}$ - A. G. E. M. de Boer ${ }^{1}$}

K. M. A. J. Tytgat

k.m.tytgat@amsterdamumc.nl

J. H. G. Klinkenbijl

j.h.g.klinkenbijl@gelre.nl

M. H. W. Frings-Dresen

m.frings@amsterdamumc.nl

A. G. E. M. de Boer

a.g.deboer@amsterdamumc.nl

1 Amsterdam UMC (Location AMC), Coronel Institute of Occupational Health, Amsterdam Public Health Research Institute, University of Amsterdam, Amsterdam, The Netherlands

2 Amsterdam UMC (Location AMC), Department of Gastroenterology, University of Amsterdam, Amsterdam, The Netherlands

3 Department of Surgery, Gelre Hospitals, Apeldoorn, The Netherlands

4 University of Amsterdam, Amsterdam, The Netherlands

5 Department of Surgery, Zaans Medical Center, Zaandam, The Netherlands

6 Department of Gastroenterology, Meander Medical Center, Amersfoort, The Netherlands

7 IKA- Ned, Hilversum, The Netherlands

8 Department of Surgery, Northwest Hospital Group, Alkmaar, The Netherlands
9 Department of Surgical Oncology, University Medical Center Groningen (UMCG), Groningen, The Netherlands

10 Department of Surgery, Albert Schweitzer Hospital, Dordrecht, The Netherlands

11 Department of Medical Oncology, Amphia Hospital, Breda, The Netherlands

12 Department of Surgery, OLVG (Location East), Amsterdam, The Netherlands

13 Tomas Rejda Counselling (Oncological Occupational Physician), Alphen aan den Rijn, The Netherlands

14 Department of Surgery, Catharina Hospital Eindhoven, Eindhoven, The Netherlands

15 Amsterdam UMC (Location AMC), Department of Surgery, University of Amsterdam, Amsterdam, The Netherlands

16 Department of Surgery, Dijklander Hospital, Hoorn, The Netherlands

17 Department of Surgery, Maastricht University Medical Center (MUMC), Maastricht, The Netherlands

18 Department of Gastroenterology, Martini Hospital, Groningen, The Netherlands

19 Department of General Surgery, Flevo Hospital, Almere, The Netherlands

20 Vel.Onc@Work Counselling (Oncological Occupational Physician), Leidschendam, The Netherlands 